\title{
Fundamental nature of the fine-structure constant
}

\author{
Michael A. Sherbon \\ Case Western Reserve University Alumnus, USA \\ E-mail: michael.sherbon@case.edu
}

Copyright (C)2014 Michael A. Sherbon. This is an open access article distributed under the Creative Commons Attribution License, which permits unrestricted use, distribution, and reproduction in any medium, provided the original work is properly cited.

\begin{abstract}
Arnold Sommerfeld introduced the fine-structure constant that determines the strength of the electromagnetic interaction. Following Sommerfeld, Wolfgang Pauli left several clues to calculating the fine-structure constant with his research on Johannes Kepler's view of nature and Pythagorean geometry. The Laplace limit of Kepler's equation in classical mechanics, the Bohr-Sommerfeld model of the hydrogen atom and Julian Schwinger's research enable a calculation of the electron magnetic moment anomaly. Considerations of fundamental lengths such as the charge radius of the proton and mass ratios suggest some further foundational interpretations of quantum electrodynamics.
\end{abstract}

Keywords: Euler's constant, Fine-structure constant, Fundamental constants, Kepler's equation, Natural philosophy.

\section{Introduction}

In addition to introducing the fine-structure constant [1]-[5], Arnold Sommerfeld added elliptic orbits to Bohr's atomic model deriving the Bohr-Sommerfeld model [6,7]. Then Wolfgang Pauli was influenced by Sommerfeld's search for the Platonic connections that were implied by the mystery of the fine-structure constant $[2,8,9]$. As the fine-structure constant determines the electromagnetic strength its theoretical origin was for Pauli a key unsolved physical problem, also considered especially significant by Max Born, Richard Feynman and many other physicists [10].

The fine-structure constant, alpha, has a variety of physical interpretations from which various determinations have been made; atom interferometry and Bloch oscillations, the neutron Compton wavelength measurement, AC Josephson effect, quantum Hall effect in condensed matter physics [11], hydrogen and muonium hyperfine structure, precision measurements of helium fine-structure, absorption of light in graphene [12], also the topological phenomena in condensed matter physics [13], the relative optical transparency of a plasmonic system [14], elementary particle lifetimes [15] and the anomalous magnetic moment of the electron in quantum electrodynamics [16]. Slightly different values of the fine-structure constant are found from different experimental measurements [17] and finally there is also the question of its variation from the subatomic to the cosmological scale [18]. The fine-structure constant is viewed in this work from both a mathematical and a physical perspective, as Hemmo and Hagar maintain that "... in current spacetime physics there can be no dynamical derivation of primitive geometrical notions such as length." In several cases, "... geometrical notions are assumed rather than derived." [19].

Max Born considered the idea of a fundamental length to be associated with the mystery of the fine-structure 
constant, see Eq. (25) [20]. The "primacy of geometry" is a mathematical assumption made in the work that follows, with the aim of progress toward additional physical understanding of the fine-structure constant [21]-[23]. Relevant to this, Wolfgang Pauli's World Clock involved basic geometric constructions depicting the cycles of time. His World Clock was likened to Kepler's first geometrical ordering of the solar system found in ancient geometry [8] and related to geometric constructions involving the Pythagorean right triangle [24].

\section{Calculating the fine-structure constant}

The fine-structure constant was proposed by Sommerfeld as the ratio of the speed of the electron in the ground state of Bohr's hydrogen atom model to the speed of light [5]:

$\alpha=v / c=e^{2} / \hbar c$,

with the elementary charge $e$, Planck's constant $h=2 \pi \hbar$ and the speed of light $c$ in cgs units; where the Coulomb constant or the permittivity factor (found in the fine-structure formula with SI units) is one and dimensionless.

An interpretation of the fine-structure constant related to Wolfgang Pauli's World Clock geometry is the standard perspective of action, product of energy and time. Two elementary particles separated by a distance $r$ have an electrostatic energy of $e^{2} / r$ and the time for light to travel a distance $r$ is $r / c$, so the action is $\left(e^{2} / r\right) \times(r / c)=e^{2} / c$. Since the unit of quantum action for light is $\hbar$, (from $E=\hbar \omega$ ), the ratio of the electrical action to the quantum action is then $e^{2} / \hbar c=\alpha$.

The fine structure constant is in the formula for the energy levels of the hydrogen atom first given by Sommerfeld. Before the discovery of the Schroedinger equation, Pauli applied the elliptical eccentricity invariant from the classical Kepler problem with the "Pauli vector" (Laplace-Runge-Lenz vector) in calculating the hydrogen spectrum [25]. In the alternative Rydberg formula for the energy levels of the hydrogen atom $E_{n}=-h c R_{\infty} / n^{2}$ with the Rydberg constant $R_{\infty}=\alpha^{2} / 2 \lambda_{e}$, having the fine-structure constant and Compton wavelength of the electron.

Julian Schwinger was noted for his introduction of $\alpha / 2 \pi$ in the corrective calculation for the anomalous magnetic moment of the electron and his zeta function regularization in the renormalization effort [26]. $\alpha / 2 \pi$ is equal to the classical electron radius divided by its Compton wavelength, see Eq. (24) discussion.

The most recent value of the inverse fine-structure constant determined by theoretical and experimental work: $\alpha^{-1}=137.035999173(35)$ in research by T. Aoyama, et al. "Tenth-Order QED Contribution to the Electron g-2 and an Improved Value of the Fine Structure Constant," [27]. Another form of calculation involves traditional Pythagorean triangles [24] together with the prime constant [28], described as a binary expansion corresponding to an indicator function for the set of prime numbers. Calculation of the inverse fine-structure constant as an approximate derivation from prime number theory:

$\alpha^{-1} \simeq 157-337 \rho / 7$

with an approximate value of $\alpha^{-1} \simeq 137.035999168$, with three prime numbers and the prime constant. The square of the diagonal of a "prime constant rectangle" is $1+\rho^{2} \simeq \kappa / e^{2} \simeq 5 / 3 \sqrt{2}$. The polygon circumscribing constant $\kappa$ is the reciprocal of the Kepler-Bouwkamp constant [29] related to "Pauli's triangle" with sides approximately proportional to $1, \phi, \sqrt{\phi \sqrt{5}}$ with the golden ratio $\phi=(1+\sqrt{5}) / 2$ [30]. $180-23=157$ and $360-23=337$. $23+37=60$ and $60 / \phi \simeq 37$ [24]. The triangles 85, 132, 157 and 175, 288, 337 are primitive Pythagorean triples. Defining $\rho$ for $\mathrm{p}(\mathrm{k})$ as the $\mathrm{k}$-th prime:

$\rho=\sum_{k=1}^{\infty} 2^{-p(k)} \simeq \phi \sqrt{5} / \kappa$

with the prime constant $\rho \simeq 0.414682509851111$ and $\kappa$ again as a reciprocal of the Kepler-Bouwkamp constant [29]. Also, $\rho \simeq \sqrt{2}-1, \phi \sqrt{5} \simeq 1+\phi^{2}=3 \phi^{3}-5 \phi^{2}+4=(5+\sqrt{5}) / 2 \simeq 3 / 2 \rho$ and $\sqrt{5}=2 \cosh (\ln \phi)$. Additionally, $\sin \alpha^{-1} \simeq 504 / 85 \kappa \approx 2 \pi / \sqrt{85}$.

$\sin \alpha^{-1} \simeq 7 ! /(713+137) \kappa$,

with the approximate value of $\alpha^{-1} \simeq 137.035999168$, same value as determined in Eq. (2) from above. Plato's favorite symbolic number $5040=7$ !. The polygon circumscribing constant, the reciprocal of the Kepler-Bouwkamp 
constant [29], is also formulated as a converging series involving the Riemann zeta function $\zeta(s)$ found in the perturbative determination of the electron magnetic moment anomaly from quantum electrodynamics.

$\kappa=\prod_{n=3}^{\infty} \sec \left(\frac{\pi}{n}\right) \simeq \phi \sqrt{5} / \rho$.

Polygon circumscribing constant $\kappa \simeq 8.700036625208$. Again approximating, $\kappa \simeq 14 / \phi$ and $\kappa^{-1} \simeq \rho^{2} \lambda$, where $\lambda$ is the Laplace limit of Kepler's equation, defined in Eq. (15).

$\phi=(1+\sqrt{5}) / 2=2 \cos (\pi / 5) \simeq \rho \kappa / \sqrt{5}$,

with the approximate value of $\phi \simeq 1.618033988749 \simeq \sqrt{2 \pi \rho}$ and $\sin \alpha^{-1} \simeq 4 / \sinh ^{2} \phi$. Also, $\sqrt{\rho k} \simeq 5 / \phi^{2} \simeq 6 / \pi \simeq$ $\sqrt[3]{7}$, which is the cube-sphere ratio (also the approximate diagonal of a golden rectangle with sides 1 and $\phi$, see references in [24]) and $\rho \kappa \simeq 3 \zeta(3) \simeq \pi R \simeq(\pi / 2) \csc (\pi / 7)$ with heptagon radius $R$ and Apery's constant $\zeta(3)$ for the value of the Riemann zeta function $\zeta(s)$, generalization of the harmonic series. Kepler showed the golden ratio $\phi[30]$ as the limit of the ratio of consecutive Fibonacci numbers [31].

$\sin \alpha^{-1} \simeq \cosh \rho \int_{0}^{\infty} \cos x^{2} d x \simeq(\kappa / 8) \sqrt{\pi / 8}$

with the Fresnel integral [32] from optical physics. From hyperbolic [33] and heptagon geometry [24], see the discussion below of Eq. (12), $\cosh \rho=\cos i \rho \simeq \kappa / 8 \simeq \sec (\pi / 7)$.

\section{Euler's constant and Kepler's equation}

The Euler-Mascheroni constant is $\gamma$, also known as Euler's constant [34]. The numerical value of Euler's constant $\gamma \simeq 0.577215664901532$. With the harmonic number $H_{n}$ :

$\gamma=\lim _{n \rightarrow \infty}\left(H_{n}-\ln n\right)=\sum_{k=2}^{\infty}(-1)^{k} \zeta(k) / k \simeq \rho \kappa / 2 \pi$.

Related approximations include $\gamma \simeq \operatorname{sech} R \simeq 4 / 7$ and $D=2 R=\csc (\pi / 7) \simeq \kappa / \pi \zeta(3) \simeq S / \sqrt{2} \simeq 4 / \sqrt{3}$, which is the approximate diameter of the circumscribing circle of the regular heptagon with side equal to one; also Apery's constant again from the Riemann zeta function $\zeta(s)$ and the silver constant $S \simeq 3.247$ [35] basic to the geometry of the regular heptagon. The heptagon radius is $R \simeq 2 \gamma \simeq \cot ^{2} \alpha^{-1}$ and $2 \rho \kappa \simeq \pi \csc (\pi / 7)$.

From optical physics, $\alpha / 2 \pi \simeq \exp (-\pi \phi n)$ where $n$ is the index of refraction for water. Snell's Law: $n_{1} \sin \theta_{1}=$ $n_{2} \sin \theta_{2}$, where $\theta_{1}$ is the angle between the ray and the surface normal in the first medium, $\theta_{2}$ is the angle between the ray and the surface normal in the second medium and $n_{1}$ and $n_{2}$ are indices of refraction $\left(n_{1} \simeq 1\right.$ in a near vacuum and $n_{2}>1$ in a transparent substance). With angle of incidence $45^{\circ}$ the angle of refraction is $32^{\circ}$ (Pauli's World Clock [24]) for water and $n_{2} \simeq 1.33 \simeq R^{2} \simeq \sqrt{7} / 2 \simeq 1 / \rho \sqrt{S} \simeq \gamma \csc (\pi / 7)$. Also approximating, $\csc \alpha^{-1} \simeq \sqrt{\phi n}$, $S \simeq 1+\sqrt{\pi \phi}$ and $\phi \pi^{2} \simeq S \ln \left(\alpha^{-1}\right)$.

$\sin \alpha^{-1} \simeq 2 \gamma \sin (\pi / 5) \simeq 2 / \gamma \pi \phi$

relating the golden ratio and inverse fine-structure constant. Other approximations, $\sin \alpha^{-1} \simeq \pi / 2 D \simeq(\pi-1) / \pi \simeq$ $\gamma \sec 32^{\circ}$. Also, $2 \cos (\pi / 7)=\sqrt{S}$ and $\csc (2 \pi / 7) \simeq \sqrt{\phi}$.

$\sin \alpha^{-1} \simeq \sqrt{2} \tan (\pi / 7) \simeq 1 / 2 \gamma \sqrt{\phi}$

The eccentricity of a golden ellipse [36] is $\varepsilon=1 / \sqrt{\phi} \simeq \csc \alpha^{-1}-\sin \alpha^{-1} \approx \mu \lambda$, see Eq. (14). The constant $\mu$ is the real fixed point of the hyperbolic cotangent and $\lambda$ is the Laplace limit of Kepler's equation [37]. Kepler's equation for calculating orbits: $M=E-\varepsilon \sin E$, where $M$ is the mean anomaly, $E$ is the eccentric anomaly and $\varepsilon$ is the eccentricity [38]-[42]. With suggestive elliptic connections from the study of polarized light, Tse and MacDonald [43], in their theory of "magneto-optical Faraday and Kerr effects of thin topological insulator films," find a "Faraday angle equal to the fine structure constant" and an approximate $\pi / 2$ Kerr rotation $[44,45]$. If the eccentricity $\varepsilon=\lambda$, which is the Laplace limit of Kepler's equation, and $E=\tan ^{-1}\left(\alpha^{-1}\right) \simeq \pi / 2$ in the parametric form of Kepler's equation with radians; then the mean anomaly $M$ is:

$M=E-\lambda \sin E \simeq E \gamma \simeq 2 \pi / 7$. 
$M \simeq \sqrt{S} / 2 \simeq 2 \sqrt{2} / \pi \simeq \pi D / 8 \simeq \cos (\pi / 7)$ and the $\sec (2 \pi / 7) \simeq \phi[24]$. From Kepler's triangle $(1, \sqrt{\phi}, \phi)$ and the heptagon, $\tan (2 \pi / 7) \simeq \sqrt{\pi / 2}$ and $\tan ^{-1}(\sqrt{\phi})$ is an approximate heptagon angle $2 \pi / 7$, with $\sqrt{\phi} \simeq 4 / \pi$. The diameter of the circumscribing sphere of the regular dodecahedron with side equal to one is $\rho \kappa \simeq 2 \pi \gamma$. Also, the outer radius of the dodecahedron $(\sqrt{3}+\sqrt{15}) / 4 \simeq \gamma / \rho$ and $\csc \alpha^{-1} \simeq R \sqrt{\phi}$. The square root of phi is described by the dodecahedron proportions. The silver constant from the heptagon:

$S=2+2 \cos (2 \pi / 7)=4 \cos ^{2}(\pi / 7) \simeq \pi \phi / e \gamma$.

The regular heptagon is related to the origin of calculus, the cycloid curve, the least action principle and the squaring of the circle; see the historical references in [24]. Silver constant $S \simeq 2 / \lambda \gamma \phi \simeq 2 \mu / \gamma \sqrt{\phi} \simeq \sqrt{\phi} \operatorname{coth} \rho \simeq$ $\operatorname{coth} \pi^{-1} \simeq \sqrt{2} D$. The real fixed point of the hyperbolic cotangent is $\mu$ and $\gamma \simeq \mu / \lambda \pi$ [46], with the Laplace limit of Kepler's equation again, see Eq. (14). The $\cosh \rho \simeq \sec (\pi / 7)=2 / \sqrt{S}$. In another reference relating to relevant geometry Li, Ji and Cao discovered that Fibonacci spirals found on conical patterns in nature can be effectively modeled as a least energy configuration [47].

\section{Electron magnetic moment anomaly}

In quantum electrodynamics the g-factor of the electron is represented in theory as a series expansion in powers of $\alpha / 2 \pi$, with the latest experimental value by the Gabrielse Research Group: $g / 2 \simeq 1.00115965218073(28)$ [48, 49]. Anomalous magnetic moment:

$a_{e}^{-1}=2 /(g-2) \simeq 26\left(34-\mu^{-1}\right)$,

approximating the value of $a_{e} \simeq 0.00115965218069$ and the real fixed point of the hyperbolic cotangent $\mu \simeq$ 1.199678640 255 773. $26 / 34=13 / 17 \simeq 2 \mu / \pi \simeq 2 / \phi^{2} .60 / 26 \simeq D, 60 / 34 \simeq 2 / R$. With $\mathrm{g} / 2$ again recalling the work of Schwinger and others [24, 26, 50].

$\mu=\operatorname{coth} \mu=\sqrt{\lambda^{2}+1} \simeq \lambda \sqrt{S} \simeq \pi / \phi^{2}$.

Laplace limit of Kepler's equation $\lambda \simeq 0.662743419349181$ and " $\ldots$ the Laplace limit is the maximum value of the eccentricity for which the series solution to Kepler's equation converges." [37]. The $\sin \alpha^{-1} \approx \mu^{-2}, \mu \approx \sqrt[12]{\kappa}$, $\lambda \approx \sqrt[12]{\alpha}, \alpha \simeq \lambda / \mu \kappa^{2}$ and $\mu \simeq \kappa / \pi D$; see the Pythagorean references in [24]. Also, $\mu / \lambda \simeq \sqrt{S}$ and $S \simeq \sqrt{2} D$. $\phi^{-2} \simeq \gamma \lambda$ and $\mu \simeq \cosh \left(\phi^{-1}\right) \simeq \cosh (\pi / 5) \simeq 6 / \pi \phi \simeq D S / 2 \pi$, see Eq. (6) discussion. The coth $x$ also appears in the Brillouin function and the Langevin function, $L(x)=\operatorname{coth} x-x^{-1}$, in the statistical mechanics of magnetic moments [51]. $L(\mu) \simeq R / \pi \simeq \mu / S \simeq 1 / e . R$ is the radius of the regular heptagon with side equal to one. The golden rhombus has angles similar to Pauli's World Clock geometry. The golden rhombus of unit edge length has long diagonal length $2 \phi / \sqrt{\phi \sqrt{5}} \simeq D^{2} / \pi \simeq \sqrt{2} \mu \simeq(8 / 9)(6 / \pi)$, with the squared-circle ratio.

$\lambda=\operatorname{csch} \mu=\sqrt{\mu^{2}-1} \simeq \mu / \sqrt{S} \simeq 1 / \gamma \phi^{2}$,

showing the Laplace limit of Kepler's equation as the hyperbolic cosecant [52] of $\mu$. $\lambda \simeq \gamma R \simeq \sinh (\pi / 5) \simeq$ $\rho \phi \simeq \gamma \cosh (1 / \sqrt{\pi}) . \quad \mu \simeq \zeta(3) \simeq \gamma / \ln \phi \simeq \pi / \phi^{2} \simeq \pi \gamma \lambda \simeq \rho \kappa \gamma^{2} \simeq \lambda \csc (\pi / 7)$ and $\rho \kappa \simeq 2 \mu / \lambda$. The $7 / 5$ ratio, relevant to Pauli's World Clock geometry, is the approximate outer radius of the dodecahedron. The golden ratio, $\phi \simeq 7 \pi / 5 e \simeq 5 e / 7 \mu$ and $\mu \simeq S \tanh (e / 7) \simeq S / e$, see Eq. (24) discussion. Also, the $\sinh \phi \simeq 5 \phi_{f} / 7 \simeq 2 \mu$ and $\phi_{f}$ is the reciprocal Fibonacci constant [53], see the parabolic constant following Eq. (24).

The Laplace limit of Kepler's equation and a 5 th degree polynomial having coefficients with a factor of 5 also gives another good approximation for the fine-structure constant:

$\alpha^{-1} \simeq \eta \pi \lambda$, where $\eta$ is a root of

$x^{5}-65 x^{4}-55 x^{3}+80 x^{2}-5 x+40=0$.

The value for $\alpha^{-1} \simeq 137.035999168$ and root $\eta \simeq 65.8171956519$ for Eq. (16). The eccentricity of a golden ellipse $\varepsilon=1 / \sqrt{\phi} \simeq \sin (2 \pi / 7) \approx \mu \lambda$, see discussion of Eq. (10). $\mu^{-1} \simeq 2 \rho \simeq 2 / \sqrt{\kappa \lambda}$ and $\rho \phi \approx \lambda$. Also, $\lambda^{-2} \simeq 1+\sqrt{\phi} \simeq$ $\sqrt{\pi \phi} \simeq \phi^{2} / R$. With $x=\alpha / 2 \pi$ :

$g / 2 \simeq 1+x-(\phi x / \sqrt{2})^{2}+(x / \gamma)^{3}-(\lambda x)^{4}+(\phi x)^{5}$, 
with alpha value from Eq. (2), $g / 2 \simeq 1.00115965218069 . \phi / \sqrt{2} \simeq 3 / L \simeq 3 \mu / \pi \simeq \ln \pi$ where $L$ is the lemniscate constant [54]. $L \simeq \pi / \mu \simeq 8 \sqrt{\kappa} / 9$, the $8 / 9$ ratio relates to the classical construction of squaring the circle; see Eq. (14) discussion and references in [24]. Also, $\lambda \simeq 1 / \mu \sqrt{\phi}$. Eq. (18) involves a convergence of Julian Schwinger's work with Green's functions [26]. Julian Schwinger introduced $\alpha / 2 \pi$ to the problem of the anomalous magnetic moment of the electron in quantum electrodynamics [26]. In the dodecahedron related to Pauli's World Clock geometry, from the center to the mid-edge the mid-radius equals $\phi^{2} / 2=(3+\sqrt{5}) / 4 \simeq 34 / 26 \simeq \pi \rho$ [55], see Eq. (6). The silver constant $S=2+2 \cos (2 \pi / 7) \simeq 1+\sqrt{\pi \phi} \simeq \sqrt{2} D \simeq 2 L / \phi \simeq 2 \ln (\pi \phi) \simeq e \mu$. A golden ellipse of unit length minor radius and major radius of length $\phi$ has an area equal to $\pi \phi[36]$ and the $\sin \alpha^{-1} \simeq 6 \gamma / \pi \phi$ with the cube-sphere ratio, see the discussion of Eq. (6).

$\operatorname{coth} \alpha \simeq \tau$, where $\tau$ is a root of

$x^{4}-137 x^{3}-6 x^{2}-98 x+343=0$,

also gives the value for $\alpha^{-1} \simeq 137.035999168$ with Eq. (19) and the value for $\tau \simeq 137.038431610$. The factor of 7 also appears as $2 \times 7^{2}=98$ and $7^{3}=343$. Eq. (20) represents a particular quartic plane curve, different combinations of the coefficients of the general curve give rise to the lemniscate of Bernoulli. Gauss's and Euler's study of the arc length of Bernoulli's lemniscate, a polar curve having the general form of a toric section, led to later work on elliptic functions. Some forms for Gauss's constant [56]:

$G=L / \pi=\frac{2}{\pi} \int_{0}^{1}\left(1-x^{4}\right)^{-\frac{1}{2}} d x=\int_{0}^{\infty}(\cosh (\pi x))^{-\frac{1}{2}} d x$.

Lemniscate constant $L=\pi G \simeq 2.622057554292119 \simeq \cosh \phi$ and $G \simeq 0.8346$ is Gauss's constant, the reciprocal of the arithmetic-geometric mean of 1 and $\sqrt{2}$, the basis for his exploration of the lemniscate function [54]. Gauss's constant is also linked with the beta function, the gamma function at argument $1 / 4$ and Jacobi theta functions. $G^{-1} \simeq \mu \simeq \lambda \sqrt{S} \simeq \pi \phi / 3 \sqrt{2}$, see Eq. (14) above. The $\sin \alpha^{-1} \simeq D_{i} G . D_{i}=\sqrt{6} / 3$, the diameter of the inscribing sphere of the octahedron. The lemniscate, inverse curve of the hyperbola with respect to its center, has the lemniscate constant $L$ which functions like $\pi$ does for the circle [54]. The lemniscate $L \simeq S \phi / 2 \simeq \cosh \phi \approx 1 / \gamma \lambda \approx$ $\phi^{2}$. With coefficients $3 / L, L \lambda, 5 / L$ and $S$ in place of $\phi / \sqrt{2}, 1 / \gamma, \lambda$ and $\phi$ there is for $x=\alpha / 2 \pi$ :

$g / 2 \simeq 1+x-(3 x / L)^{2}+(L \lambda x)^{3}-(5 x / L)^{4}+(S x)^{5}$.

With alpha value from Eq. (2), $g / 2 \simeq 1.00115965218069$. Also, $\rho \kappa \simeq L+1$, see Eq. (8).

\section{Ratios of fundamental physics}

Some fundamental mass ratios have approximations with hyperbolic functions. The $80.4 \mathrm{GeV} \mathrm{W}$ boson from electroweak theory and 125-126 GeV Higgs boson [57], $m_{w} / m_{H^{\circ}} \simeq \nu / \mu \simeq 2 / \pi$, see Eq. (28). The fixed point of the hyperbolic secant $\nu=\operatorname{sech} \nu$, Eq. (27). Higgs boson and the $136.5 \mathrm{GeV}$ Higgs-like resonance [58], $m_{H^{\circ}} / m_{H^{*}} \simeq \nu \mu \simeq$ $1 / \sqrt{\mu}$. The $91.2 \mathrm{GeV} \mathrm{Z}$ boson from electroweak theory and Higgs boson, $m_{z} / m_{H^{\circ}} \simeq(\lambda+1 / 2) / \phi$.

$m_{H^{\circ}} / m_{z} \simeq m_{t} / m_{H^{\circ}} \simeq \kappa / 2 \pi$.

Again, $\kappa$ is the reciprocal of the Kepler-Bouwkamp constant [29], see Eq. (5). Also, the Higgs boson and 172.9 $\mathrm{GeV}$ top quark from quantum chromodynamics, $m_{t} / m_{H^{\circ}} \simeq \pi \lambda^{2} \simeq \sqrt{6 / \pi} \simeq \gamma / \rho \simeq 7 / \pi \phi \simeq \sqrt{\mu \phi} \simeq \mu R \simeq \rho \kappa / \phi^{2} \simeq$ $1+\phi^{-2}$, see Eq. (6) discussion and Eq. (8). The Weinberg angle of the electroweak interaction $\cos \theta_{W}=m_{w} / m_{z} \simeq$ $\sqrt{\phi} / \mu^{2}$.

The proton/electron mass ratio [59]: $m_{p} / m_{e} \approx \exp (2 \pi \mu)$ and $2 \mu \simeq 2 \pi / \phi^{2}$ with the golden angle in radians [60, 61]. $m_{p} / m_{e} \simeq \exp (\sigma \pi) / \alpha \simeq 1836.15267237$ where $\sigma \simeq 0.8260727439178$ is a root of $13 x^{3}+28 x^{2}+37 x-57$. This is compared to the latest determination of $m_{p} / m_{e}=1836.15267245$ (75)[59]. Also, $\sigma \approx \mu^{-1}$ and $\sigma^{2} \approx \sin \alpha^{-1}$. Suggesting a squared-circle for classical electron radius/charge radius of the proton [62]:

$r_{e} / r_{p} \simeq S \sec (1 / 4)$.

Radius $r_{e}=\alpha \lambda_{e} / 2 \pi \simeq 2.81794 \times 10^{-15} \mathrm{~m}$, where $\lambda_{e} \simeq 2.4263 \times 10^{-12} \mathrm{~m}$ is the Compton wavelength of the electron. Eq. (24) gives $r_{p} \simeq 0.84089 \mathrm{fm}$ and the latest experimental result is $r_{p} \simeq 0.84087(39) \mathrm{fm}$ [62]. The ratio $r_{e} / r_{p} \simeq$ $\pi D S / 7, \pi D$ is the circumference of a regular heptagon with side equal to one and $D=2 R$. The ratio $r_{e} / r_{p} \simeq$ 
$\operatorname{coth} S^{-1} \simeq 7 \mu / \sqrt{2 \pi} \simeq \pi / \sqrt{2} \lambda \simeq \lambda \pi \phi$. Also, $R \simeq L / \sqrt{\pi \phi} \simeq 5 \phi / 7 \simeq \pi / e$, another approximation with Bernoulli's lemniscate constant. Other suggestive numerical coincidences $r_{p} / r_{e} \simeq \delta_{s} / 5 \phi \simeq \tanh S^{-1} \simeq \tan ^{2}(1 / 2)$, where $\delta_{s}$ is the silver ratio [63]. $\delta_{s}=1+\sqrt{2}=\cot (\pi / 8) \simeq 2 \mu$ and $\cosh \left(\ln \delta_{s}\right)=\sqrt{2}$. The silver ratio is the inner diameter of a regular octagon with side equal to one. The $\ln \delta_{s} \simeq \sqrt{\phi} / \mu^{2}$. The universal parabolic constant [64] $P=\sqrt{2}+\ln \delta_{s} \simeq S / \sqrt{2}$ and $\sin \alpha^{-1} \simeq P / \phi_{f}$, where $\phi_{f}$ is the reciprocal Fibonacci constant [53]. Also, $\phi_{f} \simeq \lambda \pi \phi$ and $\ln \left(\alpha^{-1}\right) \simeq S \sqrt{P}$. The $\ln \delta_{s}$ is an inflection point of $\operatorname{sech} x$, the hyperbolic secant function [65], which is a special case of the Jacobi elliptic function.

The fundamental length $g_{M}$ is from the work of Mendel Sachs [66] on the spinor-quaternion formulation for the wave function of the electron in hydrogen and prediction for the Lamb shift of the hydrogen spectrum. Sachs presents another viewpoint on the anomalous magnetic moment of the electron, developing a continuous field concept without assuming point charges and their problems with infinities. The explanation has some correlations with the golden ratio geometry of Wolfgang Pauli's World Clock [24].

$g_{M} / r_{e} \simeq 2 \pi \phi \alpha$.

$g_{M} / \lambda_{e} \simeq \phi \alpha^{2}$ and $g_{M} \simeq 2.087 \times 10^{-16} \mathrm{~m}$. Also, $g_{M} / r_{e} \simeq 2 \pi / 85$ and the $\sin \alpha^{-1} \approx 2 \pi / \sqrt{85}$.

The omega constant [67] is defined as Lambert $W(1)\left[[68,69]\right.$, the attractive fixed point of $e^{-x}: W(1)=\Omega=$ $\exp (-\Omega) \simeq 0.567 \simeq 1 / \sqrt{\pi} \simeq \phi^{2} / 2 D$ and $17 / 13 \simeq \pi \rho \simeq \phi^{2} / 2 \simeq D \Omega$.

$\Omega=\exp (-\Omega) \simeq \sinh ^{-1}(\mu / 2)$.

$W(x)$, the Lambert $W$-function, is an analog of the golden ratio for exponentials as $\exp [-W(1)]=W(1)$. The $\sin \alpha^{-1} \approx 6 \Omega / \pi \phi$. The $\cosh \Omega \simeq \lambda / \Omega$ and the $\operatorname{csch} \nu \simeq \mu$, where $\mu$ again is equal to the $\operatorname{coth} \mu$ and $\nu$ is the real fixed point of the hyperbolic secant:

$\nu=\operatorname{sech} \nu \simeq 1 / D \Omega \simeq \mu \sqrt{\phi} / 2$.

Again, $\operatorname{csch} \nu \simeq \mu$ and $\nu \simeq 13 / 17 \simeq \delta_{s} / \pi \simeq 1-\zeta(2) / 7 \simeq 1 / \pi \rho \simeq 2 / \phi^{2} \simeq 0.765$. With the Weinberg angle of the electroweak interaction $\cos \theta_{W}=m_{w} / m_{z} \simeq 1 / 2 \Omega \simeq \lambda / \nu$. From Adamchik's integral for the omega constant [67] the value is $(1+\Omega)^{-1} \simeq R / \sqrt{S} \simeq 0.638$.

$\nu / \mu \simeq(1+\Omega)^{-1}=\int_{-\infty}^{+\infty}\left(\left(e^{x}-x\right)^{2}+\pi^{2}\right)^{-1} d x$.

Also, $m_{w} / m_{H^{\circ}} \simeq \nu / \mu \simeq \sqrt{\phi} / 2 \simeq 2 / \pi$, an approximation with the golden ratio geometry. From the Foundation Stone of classical harmonic theory, the alpha harmonic is equal to the sum of the golden ratio harmonic and the omega constant harmonic, relating the Greek Pythagorean form of the fine-structure constant to the golden ratio geometry [24].

\section{Conclusion}

From Arnold Sommerfeld's introduction of the fine-structure constant and Wolfgang Pauli's search for an explanation, approximate values for the fine-structure constant have been determined. With an extension of the Keplerian intuition regarding the fundamental geometry of basic polygons, conic sections and Platonic polyhedra included in Wolfgang Pauli's World Clock geometry; the mathematical and physical model for the calculations is an alternative to accounting for individual contributions of the interactions between field quanta and begins to address some of the questions raised by Richard Feynman, Freeman Dyson, Paul Dirac and others about quantum electrodynamics [70].

The polygon circumscribing constant, reciprocal of the Kepler-Bouwkamp constant, has interesting geometric connections with the torus topology relating the relativity of Einstein with the geometry of classical and quantum mechanics. The nature of this topology was pursued by Wolfgang Pauli and is suggested in the Keplerian paradigm of Pythagorean harmonic proportions from ancient geometry [24]. Quantum mechanics is thus found to be an approximation theory based on the classical mathematical problem of squaring the circle, with all of its complex analogies and philosophical implications.

\section{Acknowledgements}

Special thanks to Case Western Reserve University, MathWorld and WolframAlpha. 


\section{References}

[1] Barrow, J.D. The Constants of Nature: From Alpha to Omega-the Numbers That Encode the Deepest Secrets of the Universe, New York: Pantheon Books, 2002.

[2] Miller, A.I. Deciphering the Cosmic Number, New York: W.W. Norton, 2009.

[3] Uzan, J.P. \& Leclercq, B. The Natural Laws of the Universe: Understanding Fundamental Constants, New York: Springer, 2008.

[4] Fritzsch, H. The Fundamental Constants, Hackensack, NJ: World Scientific, 2009.

[5] Sommerfeld, A. "On the Quantum Theory of Spectral Lines," Annals of Physics, 51, 1-94, 125-67 (1916).

[6] Sommerfeld, A. Atomic Structure and Spectral Lines, London: Methuen, 118, 1923.

[7] Kragh, H. Niels Bohr and the Quantum Atom, NY: Oxford University Press, 2012.

[8] Enz, C.P. No Time to be Brief: A Scientific Biography of Wolfgang Pauli, New York: Oxford University Press, 2002.

[9] Miller, A.I. Early Quantum Electrodynamics: A Source Book, Cambridge, UK: Cambridge University Press, 1995.

[10] Feynman, R.P. QED: The Strange Theory of Light and Matter, Princeton, NJ: Princeton University Press, 1985.

[11] Yoshihiro, K. et al. "Determination of the Fine Structure Constant Based on the Quantum Hall Effect," Progress of Theoretical Physics, 84, 1, 215-223 (1985).

[12] Nair, R.R. et al. "Fine Structure Constant Defines Visual Transparency of Graphene," Science, 320, 5881, 1308-1308 (2008) arXiv:0803.3718v1.

[13] Maciejko, J. et al. "Topological Quantization in Units of the Fine Structure Constant," Physical Review Letters, 105, 16, 166803 (2010) arXiv:1004.2514v3.

[14] Kravets, V.G. et al. "Fine Structure Constant and Quantized Optical Transparency of Plasmonic Nanoarrays," Nature Communications, 3, 640, (2012).

[15] Mac Gregor, M.H. The Power of Alpha, Hackensack, NJ: World Scientific, 2007.

[16] Sturm, S., Werth, G. \& Blaum, K. "Electron g-Factor Determinations in Penning Traps," Annals of Physics, 525, 8-9, 620-635 (2013).

[17] Karshenboim, S. "Recent Progress in Determination of Fundamental Constants and Fundamental Physics at Low Energies," Annals of Physics, 525, 7, 472-483 (2013).

[18] Truppe, S., Hendricks, R.J., Tokunaga, S.K., Lewandowski, H.J. Kozlov, M.G. et al. "A Search for Varying Fundamental Constants using Hertz-Level Frequency Measurements of Cold CH Molecules," Nature Communications, 4, 2600 (2013).

[19] Hagar, A. \& Hemmo, M. "The Primacy of Geometry," Studies in History and Philosophy of Modern Physics, 44, 3, 357-364 (2013).

[20] Born, M. "The Mysterious Number 137," Proceedings of the Indian Academy of Sciences - Section A, 2, 6, 533-561 (1935).

[21] Blaum, K., Muller, H. \& Severijns, N. "Precision Experiments and Fundamental Physics at Low Energies - Part I," Annals of Physics, 525, 7, A111-A112 (2013).

[22] Ubachs, W., Vassen, W. et al. "Precision Metrology on the Hydrogen Atom in Search for New Physics," Annals of Physics, 525, 7, A113-A115, (2013).

[23] Bouchendira, R. et al. "State of the Art in the Determination of the Fine Structure Constant," Annals of Physics, 525, 7, 484-492 (2013) arXiv:1309.3393v1.

[24] Sherbon, M.A. "Wolfgang Pauli and the Fine-Structure Constant," Journal of Science, 2, 3, 148-154 (2012) SSRN: 2147980 .

[25] Pauli, W. "On the Hydrogen Spectrum from the Standpoint of the New Quantum Mechanics," Journal of Physics A: Hadrons and Nuclei, 36, 5, 336-363 (1926). 
[26] Schwinger, J. "On Quantum Electrodynamics and the Magnetic Moment of the Electron," Physical Review, 73, 4, 416-417 (1948).

[27] Aoyama, T., Hayakawa, M., Kinoshita, T. \& Nio, M. "Tenth-Order QED Contribution to the Electron g-2 and an Improved Value of the Fine Structure Constant," Physical Review Letters, 109, 111807 (2012) arXiv:1205.5368v2.

[28] Weisstein, E.W. "Prime Constant," MathWorld-A Wolfram Web Resource.

[29] Finch, S.R. "Kepler-Bouwkamp Constant," Mathematical Constants, Cambridge: Cambridge University Press, 428-429, 2003.

[30] Weisstein, E.W. "Golden Ratio," MathWorld-A Wolfram Web Resource.

[31] Stakhov, A. "The Golden Section and Modern Harmony Mathematics," in Bergum, G.E. et al. Eds. Applications of Fibonacci Numbers, NY: Springer, 393-399, 1998.

[32] Weisstein, E.W. "Fresnel Integrals," MathWorld-A Wolfram Web Resource.

[33] Stakhov, A.P. \& Rozin, B.N. "The Golden Section, Fibonacci Series and New Hyperbolic Models of Nature," Visual Mathematics, 8, 3 (2006).

[34] Lagarias, J.C. "Euler's Constant: Euler's Work and Modern Developments," Bulletin of the American Mathematical Society, 50, 4, 527-628 (2013) arXiv:1303.1856.

[35] Weisstein, E.W. "Silver Constant," MathWorld-A Wolfram Web Resource.

[36] Huntley, H.E. "The Golden Ellipse," The Fibonacci Quarterly, 12, 1, 38-40 (1974).

[37] Weisstein, E.W. "Laplace Limit," MathWorld-A Wolfram Web Resource.

[38] Cariglia, M. \& Araujo, E.S. "Dynamical Symmetries of the Kepler Problem," European Journal of Physics, 34, 5, 1307 (2013) arXiv:1309.6913.

[39] Nucci, M.C. \& Leach, P.G.L. "The Harmony in the Kepler and Related Problems," Journal of Mathematical Physics, $42,746(2001)$.

[40] Rogers, H.H. "Symmetry Transformations of the Classical Kepler Problem," Journal of Mathematical Physics, 14, 1125-1129 (1973).

[41] Dahl, J.P. "Physical Origin of the Runge-Lenz Vector," Journal of Physics A: Mathematical and General, 30, 19, 6831 (1997).

[42] Wulfman, C.E. "On the Dynamical and Geometrical Symmetries of Keplerian Motion," Journal of Physics A: Mathematical and Theoretical, 42, 18, 185301 (2009).

[43] Tse, W.K. \& MacDonald, A.H. "Magneto-Optical Faraday and Kerr Effects in Topological Insulator Films and in Other Layered Quantized Hall Systems," Physical Review, B84, 205327 (2011) arXiv:1108.3858v1.

[44] Shimano, R. et al. "Quantum Faraday and Kerr Rotations in Graphene," Nature Communications, 4, 1841 (2013).

[45] Burinskii, A. "The Dirac-Kerr-Newman Electron," Gravitation and Cosmology, 14, 4, 2, 109-122 (2008) arXiv:hepth/0507109v4.

[46] Weisstein, E.W. "Hyperbolic Cotangent," MathWorld-A Wolfram Web Resource.

[47] Li, C., Ji, A. \& Cao, Z. "Stressed Fibonacci Spiral Patterns of Definite Chirality," Applied Physics Letters, 90, 164102, (2007).

[48] Hanneke, D. et al. "New Measurement of the Electron Magnetic Moment and the Fine Structure Constant," Physical Review Letters, 100, 120801 (2008).

[49] Hanneke, D. et al. "Cavity Control of a Single-Electron Quantum Cyclotron: Measuring the Electron Magnetic Moment," Physical Review, A83, 5, 052122 (2011).

[50] Pfister, H. \& King, M. "The Gyromagnetic Factor in Electrodynamics, Quantum Theory and General Relativity," Classical and Quantum Gravity, 20, 1, 205 (2003).

[51] Greiner, W., Neise, L. \& Stcker, H. Thermodynamics and Statistical Mechanics, New York: Springer, $216,1997$. 
[52] Weisstein, E.W. "Hyperbolic Cosecant," MathWorld-A Wolfram Web Resource.

[53] Sloane, N.J.A. "Reciprocal Fibonacci Constant," The On-Line Encyclopedia of Integer Sequences, OEIS: A079586.

[54] Weisstein, E.W. "Lemniscate Constant," MathWorld-A Wolfram Web Resource.

[55] Heyrovska, R. "Golden Ratio Based Fine Structure Constant and Rydberg Constant for Hydrogen Spectra," International Journal of Sciences, 2, 3, 28-31 (2013).

[56] Weisstein, E.W. "Gauss's Constant," MathWorld-A Wolfram Web Resource.

[57] Beringer, J. et al. (Particle Data Group) "Review of Particle Physics - Gauge and Higgs Bosons," Physical Review, D86, 1 (2012).

[58] CMS Collaboration, "Properties of the Observed Higgs-like Resonance using the Diphoton Channel," PAS CMS HIG13-016 (2013) cds.cern.ch/record/1558930.

[59] Mohr, P.J., Taylor, B.N. \& Newell, D.B. "CODATA Recommended Values of the Fundamental Physical Constants," Reviews of Modern Physics, 84, 4, 1527 (2012).

[60] Weisstein, E.W. "Golden Angle," MathWorld-A Wolfram Web Resource.

[61] Munteanu, M.I. "From Golden Spirals to Constant Slope Surfaces," Journal of Mathematical Physics, 51, 7, 073507, 1-9 (2010) arXiv:0903.1348v1.

[62] Antognini, A. et al. "Proton Structure from the Measurement of 2S-2P Transition Frequencies of Muonic Hydrogen," Science, 339, 6118, 417-420 (2013).

[63] Weisstein, E.W. "Silver Ratio," MathWorld-A Wolfram Web Resource.

[64] Reese, S. \& Sondow, J. "Universal Parabolic Constant," The On-Line Encyclopedia of Integer Sequences, OEIS: A103710.

[65] Weisstein, E.W. "Hyperbolic Secant," MathWorld-A Wolfram Web Resource.

[66] Sachs, M. Quantum Mechanics from General Relativity, New York: Springer, 1986.

[67] Weisstein, E.W. "Omega Constant," MathWorld-A Wolfram Web Resource.

[68] Valluri, S.R, Jeffrey, D.J. \& Corless, R.M. "Some Applications of the Lambert W Function to Physics," Canadian Journal of Physics, 78, 9, 823-831 (2000).

[69] Weisstein, E.W. "Lambert W-Function," MathWorld-A Wolfram Web Resource.

[70] Schweber, S.S. QED and the Men Who Made It: Dyson, Feynman, Schwinger and Tomonaga, Princeton, NJ: Princeton University Press, 1994. 